\title{
Analysis of Adaptive Modulation with Antenna Selection under Channel Prediction Errors
}

\author{
Shiva Prakash, Student Member, IEEE, Ian McLoughlin, Senior Member, IEEE
}

\begin{abstract}
The performance of an adaptive modulation system in Rayleigh fading channels exploiting spatial diversity through transmit antenna selection is analysed for the case of delay constrained networks. The system combines maximal ratio combining at the receiver and a transmit antenna selection system which switches between available antennas and modulation schemes at the transmitter. In a practical system, a delay between the channel being sampled at the receiver and acted upon by the transmitter will tend to degrade system performance. In this paper, a channel prediction scheme is employed at the receiver to provide estimates of future best transmission states, which includes selecting the best transmission antenna as well as the best supported M-QAM modulation scheme. The Shannon capacity for optimal rate and constant power is derived and presented, and used as a benchmark to evaluate the spectral efficiency of the discrete rate system optimised for instantaneous BER (bit error rate) and constant power constraint.
\end{abstract}

\section{INTRODUCTION}

By exploiting spatial diversity, MIMO (Multiple input multiple output) technology has been shown to provide capacity gain without incurring power or bandwidth cost [1]. Further gains can be achieved by adapting to the varying channel fluctuations, by adjusting parameters such as constellation size, transmit power and code rate to match changes in the channel [2], [3], [4], [5]. However, the performance of adaptive modulated schemes is limited by CSI (channel state information) imperfections particularly in the form of delay in the feedback channel, which causes outdated channel information at the transmitter, thus reducing capacity. The use of imperfect channel estimates for SISO channels is investigated in [6], [7] while the effect of feed back delay in adaptive modulation (AM) systems over a Nakagami fading SISO has been considered in [8], showing the degradation of BER (bit error rate) with feedback delay. To mitigate the effect of feedback delay, channel prediction has been employed for use in SISO channels using a pilot symbol aided modulation (PSAM) technique [9], while [10], [11], [12], [13] consider multiple antenna schemes. This paper investigates the issues relating to degraded CSI for an un-coded TAS/MRC (transmit antenna selection with maximal ratio combing) system with prediction, which adaptively adjusts modulation format based upon outdated CSI, in order to maximise spectral efficiency at a target BER. The predicted future CSI is used at the receiver to select the best switching parameters for transmission. Although the use of a feedback channel is necessary, in practice only a low bandwidth channel is required since it is simply the indices of the best predicted antenna and rate that need to be fed back. TAS [14], is beneficial in reducing

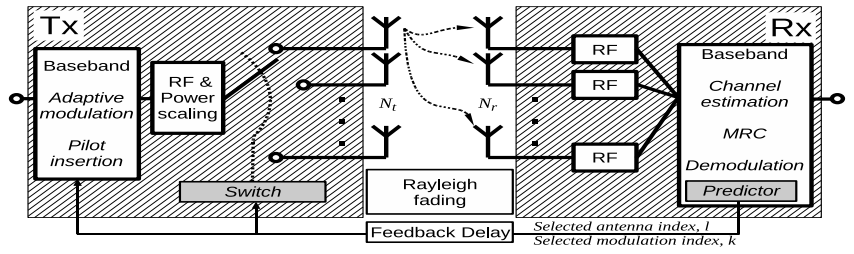

Fig. 1. A $\left(N_{t} ; 1, N_{r}\right)$ transceiver block diagram employing both adaptive modulation and transmit antenna selection with MRC.

number of transmit chains and has been shown to achieve full diversity as if all the transmit antennas were used (at high SNR), outperforming STC (space time codes) of the same spectral efficiency. The performance of non-adaptive TAS is usually limited by the quality of the channel knowledge, which is an index to the best antenna which offers the highest SNR gain amongst all transmit antennas. Large delays render the feedback information useless, effectively creating an openloop system. In [15], channel prediction was applied to a nonadaptive TASP/MRC (predicted TAS/MRC) system, where the effects due to delay is mitigated by predicting the antenna index at the receiver, several coherence intervals ahead. In this paper we consider an adaptively modulated TASP/MRC system, optimised for instantaneous BER (I-BER) constraint under constant power. In Section II, we describe the TASP system model, the channel prediction scheme and fading PDF (probability density function) of the system. In Section III, we derive the closed form expression for the Shannon capacity bound for optimal rate and constant power for the TAS/MRC scheme under prediction, and use it as a benchmark to compare our different transmission schemes. In Section IV, we analytically describe the TASP/MRC AM system. In Section V, we consider the I-BER constraint with constant power scheme, and derive optimal switching boundaries for the AM system. Section VI discusses the performance analysis in terms of average BER and spectral efficiency, comparing it with nonadaptive TASP/MRC schemes. Section VII has the conclusion. Notation used in the paper: $(.)^{H}$ stands for conjugate transpose, $E[$.$] stands for expectation, I_{P}$ stands for identity matrix of size $P$.

\section{System Model AND Assumptions}

We consider a point-to-point MIMO adaptive system as shown in Fig. 1, equipped with $N_{t}$ transmit and $N_{r}$ receive antennas. The transmitter is capable of switching between different M-QAM (M-Quadrature amplitude modulation) constellations as well as selecting a single best antenna based on 
information feedback from the receiver. This system is represented as $\left(N_{t} ; 1, N_{r}\right)$. At the receiver, channel estimates are assumed to be error-free for demodulation, so that noncausal channel smoothing with high accuracy can be performed using Wiener interpolator filters (which are non-causal) and hence near perfect demodulation can be assumed [11], [8], [12]. In an adaptive TAS/MRC system, degradation from using outdated channel estimates can occur in two ways: from a) not selecting a good antenna in the present slot b) choosing an incorrect modulation level or rate. Prediction of transmission states in advance is therefore necessary to alleviate the losses that occur because of using outdated channel estimates. Note that however, the channel predictor is strictly causal.

To enable channel prediction and estimation, known pilot symbols are transmitted [16] from each antenna in turn at different time slots into a fixed frame length $L_{f}$, and channel estimation for a frame of data is carried out independently for all channels. A block stationary and ergodic channel is assumed. The elements of $\mathbf{H}$, the $N_{r} \times N_{t}$ channel matrix, are assumed to be constant over a block, however temporal correlation is assumed across blocks. $h_{i j}(m)$ is an entry of the channel matrix $\mathbf{H}(m), 1 \leq j \leq N_{r}, 1 \leq i \leq N_{t}$, and is the true fading coefficient of the channel between the $i$ th transmit and the $j$ th receive antenna corresponding to the $m^{\text {th }}$ transmitted block. The entries of $\mathbf{H}(m)$ the are then estimated as: $\tilde{h}_{i j}(m)=h_{i j}(m)+v_{i j}(m)$. The channels $h_{i j}(m)$ are characterised as independent and identically distributed (i.i.d) complex Gaussian random variables $\mathcal{C N}\left(0, \sigma_{h}^{2}\right)$ that follow Jakes model [17] with Doppler spread $f_{d}$ and channel power $\sigma_{h}^{2}$. Correspondingly, $\tilde{h}_{i j}(m)$ is the channel estimate while $v_{i j}(m)$ is the AWGN channel estimation error with distribution $\mathcal{C N}\left(0, \sigma_{v}^{2}\right)$, and $\sigma_{v}^{2}=N_{0} / E_{p}, E_{p}$ being the power of the pilot symbol. Assuming $\sigma_{h}^{2}=1$, the variance of the estimated channel amplitude is $\sigma_{\tilde{h}}^{2}=1+\sigma_{v}^{2}$. Using these estimates, the CSI can be predicted using WienerHopf equations so that the $n$ block ahead predicted channel can be written as $\hat{h}_{i j}(m+n)=\mathbf{w}_{o p t}^{H} \widetilde{\mathbf{h}}_{i j}$ where $\widetilde{\mathbf{h}}_{i j}$ is the complex vector of estimated fading amplitudes corresponding to a prediction length $L$ given by $\tilde{\mathbf{h}}_{i j}=$ $\left[\tilde{h_{i j}}(m) \tilde{h_{i j}}(m-1), \ldots \tilde{h_{i j}}(m-(L-1)]^{T}\right.$ and $\mathbf{w}_{o p t}$ is the complex coefficient vector given by $\mathbf{w}_{o p t}=\mathbf{R}_{w}^{-1} \mathbf{r}_{w}$ where $\left[\mathbf{R}_{w}\right]_{\varphi \vartheta}=J_{o}\left(2 \pi f_{d}|\varphi-\vartheta| L_{f} T\right)+\sigma_{v}^{2} \delta(\varphi-\vartheta)$, is the covariance matrix and $T$ is the symbol period, and $\left[\mathbf{r}_{w}\right]_{\varphi}=J_{o}\left(2 \pi f_{d}|n+\varphi-1| L_{f} T\right)$ with $\varphi, \vartheta=1,2, \ldots L$. $J_{0}($.$) is the zeroth order Bessel function of the first kind.$ Here the channel delay is assumed to be an integer multiple of $L_{f}$, i.e $\tau=n L_{f} T$. The predicted channel SNR for any transmit antenna $i$ at time $(m+n)$ is then given by: $\widehat{\gamma}_{i}=\frac{E_{s}}{N_{0}} \sum_{j=1}^{N_{r}}\left|\hat{h}_{i j}\right|^{2}$ where $E_{s} / N_{0}$ is the average received SNR, depicted as $\bar{\gamma}$. Based on $\widehat{\gamma_{i}}$, the receiver will choose the appropriate good antenna, as well as the appropriate rate for the future transmission slot. Here we will discuss the fading PDF of the TASP/MRC system, useful for system analysis. A single best transmit antenna is picked from $N_{t}$ candidates, based upon the maximum SNR gain: $\widehat{\gamma}_{\max }=$ $\max _{1 \leq i \leq N_{t}}\left[\widehat{\gamma}_{i}\right]$. Besides the true maximum SNR $\gamma_{\max }$ at time corresponding to predicted maximum SNR $\widehat{\gamma}_{\max }$ is given as $\gamma_{\text {max }}=\max _{1<i<N_{t}}\left[\gamma_{i}\right]$ with the true SNR for antenna $i$ as: $\gamma_{i}=\frac{E_{s}}{N_{0}} \sum_{j=1}^{N_{r}^{-}}\left|h_{i j}\right|^{2}$. The index of the maximum SNR $\widehat{\gamma}_{\max }$ denoted as $l$ as well as the appropriate rate, is fed back to the transmitter, through a feedback channel (shown in Fig. 1). Based on the index, a single best transmitter is chosen for transmission at time instant $q=(m+n)$ and the received signal vector can be expressed as: $\mathbf{y}(q)=\mathbf{h}_{l}(q) x(q)+\mathbf{z}(q)$ where $x(q)$ represents the un-coded symbol transmitted from the single selected antenna, $\mathbf{z}(q)$ is the AWGN vector with distribution $\mathcal{C N}\left(0, \sigma_{z}^{2} \mathbf{I}_{N_{r}}\right), \mathbf{h}_{l}(q)$ is the $N_{r} \times 1$ chosen vector corresponding to the maximum power gain, which is the $l$ th column of the channel matrix $\mathbf{H}(q)$, assumed to be perfectly estimated at the receiver for demodulation with MRC. Both $\gamma_{i}$ and $\hat{\gamma}_{i}$ are gamma distributed with PDFs, $\gamma_{i} \sim G\left(N_{r}, \bar{\gamma}\right)$ and $\hat{\gamma}_{i} \sim G\left(N_{r}, \overline{\hat{\gamma}}\right)$ where and $\overline{\hat{\gamma}}=\bar{\gamma} \sigma_{\hat{h}}^{2}=\bar{\gamma} r_{w}^{H} R_{w}^{-1} r_{w}$ being the shape factors of the gamma distributions, also equal to the average power gain per symbol for the true and predicted channel respectively. The MSE for the biased predictor is $\sigma_{\epsilon_{p}}^{2}=E\left[|\gamma-\hat{\gamma}|^{2}\right] . \rho_{p}=\operatorname{Cov}(\gamma, \hat{\gamma})[\operatorname{Var}(\gamma) \operatorname{Var}(\hat{\gamma})]^{-0.5}$, is the power channel correlation coefficient and can be shown to be equal to the square of the channel correlation coefficient, when a Wiener filter is used for prediction, considering second order statistics in a stationary channel modelled using the Jakes' Doppler spectrum. In [15], we derive the PDF: $f_{\gamma_{T A S P}}(\gamma)$, which is equivalent to the the PDF of the true maximum SNR, $\gamma_{\max }$ obtained by averaging out the conditional PDF $f(\gamma \mid \hat{\gamma})$ a bivariate Gamma function [15], with PDF of $f_{\hat{\gamma}_{\max }}(\hat{\gamma})$. This is given as:

$$
f_{\gamma_{T A S P}}(\gamma)=\int_{0}^{\infty} f(\gamma \mid \hat{\gamma}) f_{\hat{\gamma}_{\max }}(\hat{\gamma}) d \gamma
$$

where the PDF $f_{\hat{\gamma}_{\max }}(\hat{\gamma})$ can be calculated as follows:

$$
f_{\hat{\gamma}_{\text {max }}}(\hat{\gamma})=N_{t} f(\hat{\gamma})[F(\hat{\gamma})]^{N_{t}-1}
$$

\section{Maximum Achievable Spectral EfFiciency}

The average spectral efficiency of a wireless system denotes the average information rate or capacity per unit bandwidth. One technique to improve this is through adaptive coding and modulation (ACM) [6]. In this paper we consider prediction benefits with rate adaptation and un-coded M-QAM modulation, although the approach in this paper can be extended for coding schemes. To analyse adaptive transmission in a transmit antenna selection-with-prediction (TASP) system, we are motivated to derive the Shannon capacity denoted as $<C_{\text {oratas }}>$ [bits/s], with optimal rate adaptation under constant power. The maximum average spectral efficiency in bits/sec/Hz in a channel of bandwidth $W$ of the TASP system is obtained as [8]:

$$
<C_{\text {oratas }}>/ W=\int_{0}^{\infty} \log _{2}(1+\gamma) f_{\gamma_{T A S P}}(\gamma) d \gamma
$$

Using (1) and [eqn.(32) of [18]], the and the expansion for the expression for $<C_{\text {oratas }}>/ W$ under imperfect prediction 
can be shown to be:

$$
\begin{gathered}
<C_{\text {oratas }}>/ W=\frac{N_{t} !}{\left(N_{r}-1\right) !} \sum_{i=0}^{N_{t}-1} \frac{(-1)^{i}}{i !\left(N_{t}-1-i\right) !} \\
\exp [-\mu] \sum_{j=0}^{i\left(N_{r}-1\right)} \eta\left(i, j, N_{r}\right)\left(j+N_{r}-1\right) ! \sum_{k=0}^{j}\left(\frac{1}{\bar{\gamma}}\right)^{j+N_{r}} \\
\frac{\rho_{p}^{k}\left(1-\rho_{p}\right)^{j-k}}{\left[i\left(1-\rho_{p}\right)+1\right]^{j+k+N_{r}}} \sum_{l=1}^{k+L_{r}} \Gamma\left[-k-N_{r}+l, \mu\right] / \mu !
\end{gathered}
$$

where $\eta_{N_{r}}(i, j)$ [19] are the coefficients of $z^{j}, j=$ $0,1, \ldots i\left(N_{r}-1\right)$, in the expansion of $\left(\sum_{j=0}^{N_{r}-1} z^{j}\right)^{i}$ and $\mu=$ $(i+1) /\left(\bar{\gamma} i\left(1-\rho_{p}\right)+1\right) . \Gamma(-n, x)$ is incomplete gamma function. By setting $\rho_{p}=1$, the maximum achievable spectral efficiency can be obtained for different TAS/MRC configurations. Fig. 2 and reveal how the system behaves for varying number of antennas, and different degrees of fading correlation. Fig.2a shows two sets of curves for $\rho_{p}=1$, where the number of transmit antennas are increased while the number of receive antennas are kept constant. It is observed that increasing the number of transmit antennas yields diminishing capacity gains when the number of receive antennas is large. Fig. 2b, plotted for $\rho_{p}=0.7$, shows that the capacity gains when compared to Fig. 2 are reduced. Also the effect of increasing $N_{t}$ is further reduced when compared to the perfectly predicted case. The capacity of the $\left(1 ; 1, N_{r}\right)$ case, with only receive diversity, acts as a lower bound to the capacity of the TASP/MRC schemes, since when CSI is completely outdated, i.e for a case when $\rho_{p}=0$, the TASP/MRC tends to a simple MRC system.

\section{System ANALYSis}

In an adaptive discrete rate (ADR) modulated scheme, the modulation mode is changed based on the instantaneous channel quality measure, which in this case is the predicted SNR value for the best transmit antenna selected for transmission in a future slot. In a K-mode modulated scheme, the transmit mode $k \epsilon\{0,1, \ldots K-1\}$ with throughput $R_{k}=\log _{2}\left(M_{k}\right)$ and constellation size $M_{k} \neq 0$, is chosen when $\hat{\gamma}_{k} \leq \hat{\gamma}<\hat{\gamma}_{k+1}$, where the switching level $\hat{\gamma}_{k}$ belongs to set $v=\left\{\hat{\gamma}_{k} \mid k=\right.$ $0,1, \ldots K\} . R_{0}=0$ when there is no transmission [20]. $\hat{\gamma}_{1}$ will be the cut off SNR below which there will be no transmission with $\hat{\gamma}_{0}=0$ and $\hat{\gamma}_{K}=\infty$. Here we will discuss the derivations of important performance measures namely average throughput, conditional BER, average BER and mode selection probability; these will be used to obtain optimal operating conditions for maximum performance under given channel prediction errors. The spectral efficiency $S E$ or average throughput $B$ is given as: $B=\sum_{k=0}^{K-1} R_{k} P_{k}$, where $P_{k}$ is the probability of mode selection given by:

$$
P_{k}=\int_{\hat{\gamma}_{k}}^{\hat{\gamma}_{k+1}} f_{\hat{\gamma}_{\max }}(\hat{\gamma}) d \hat{\gamma}=F_{\hat{\gamma}_{\max }}\left(\hat{\gamma}_{k+1}\right)-F_{\hat{\gamma}_{\max }}\left(\hat{\gamma}_{k}\right)
$$

where $F_{\hat{\gamma}_{\max }}(\hat{\gamma})$ is the CDF of $f_{\hat{\gamma}_{\max }}(\hat{\gamma})$. Considering that the receiver has no knowledge of $\gamma$, we can then find the average instantaneous BER conditioned on $\hat{\gamma}$ (which can be thought of

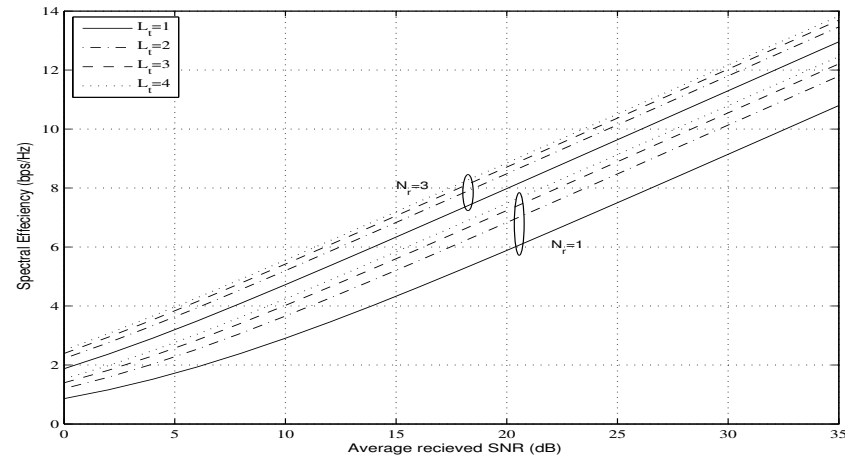

(a)

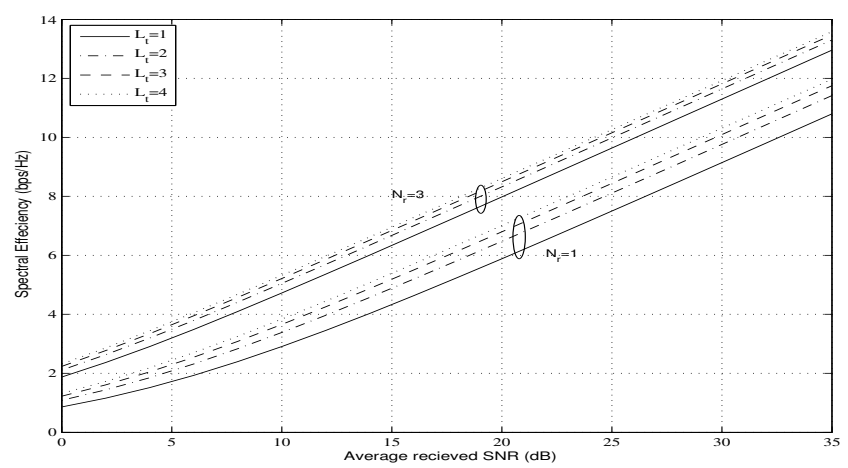

(b)

Fig. 2. Maximum achievable spectral efficiency for different TAS configurations $\left(N_{t} ; 1, N_{r}\right)$ with (a) perfect prediction or zero delay case: $\rho_{p}=1$ and (b) imperfect prediction: $\rho_{p}=0.7$.

as a short term average) by averaging the actual instantaneous BER, $B E R(\gamma \mid \hat{\gamma})$ over all values of the $\gamma$ :

$$
\operatorname{BER}(\hat{\gamma})=\int_{0}^{\infty} \operatorname{BER}(\gamma \mid \hat{\gamma}) f(\gamma \mid \hat{\gamma}) d \gamma
$$

where

$$
\operatorname{BER}(\gamma \mid \hat{\gamma}) \approx 0.2 \exp \left(-\frac{1.6 \gamma}{M_{k}(\hat{\gamma})-1} P(\hat{\gamma})\right)
$$

which [21] is approximate invertible expression for the instantaneous BER over the selected antenna as a function of $\gamma$ and transmit power $P(\hat{\gamma})$, which is accurate for $M_{k}(\hat{\gamma}) \geq 4$ and BER $\leq 10^{-2}$. Solving (6) we get:

$$
\operatorname{BER}(\hat{\gamma})=0.2\left(1+C_{k}\right)^{-L_{r}} \exp \left(-\frac{1.6 P \hat{\gamma} /\left(M_{k}-1\right)}{1+C_{k} P}\right)
$$

where $\mathcal{C}_{k}=1.6 \bar{\gamma}\left(1-\rho_{p}\right) /\left(M_{k}-1\right)$. This will be adapted for the case of constant transmit power. $P(\hat{\gamma})=P$, a constant dependent on $\hat{\gamma}_{1}$ to be optimised later in Section V (with constraint of an average power $\bar{P}=1$ ). The average BER is the ratio between the average number of bits in error and the total number of transmitted bits [7], [8] given as:

$$
B E R_{a v}=\frac{\sum_{k=1}^{K-1} B E R_{k} R_{k}}{\sum_{k=1}^{K-1} P_{k} R_{k}}
$$


Combining (8) and (2), a closed form solution of $B E R_{k}$ which is the the mode specific bit error rate can be found as:

$$
\begin{aligned}
B E R_{k} & =\int_{\hat{\gamma}_{k}}^{\hat{\gamma}_{k+1}} \int_{0}^{\infty} \operatorname{BER}(\gamma \mid \hat{\gamma}) f(\gamma \mid \hat{\gamma}) f_{\hat{\gamma}_{\max }}(\hat{\gamma}) d \gamma d \hat{\gamma} \\
& =\int_{\hat{\gamma}_{k}}^{\hat{\gamma}_{k+1}} \operatorname{BER}(\hat{\gamma}) f_{\hat{\gamma}_{\max }}(\hat{\gamma}) d \hat{\gamma}
\end{aligned}
$$

Finally average BER (9) can be computed using (5) and (10). The BER curves of the fixed-mode, non-adaptive M-QAM with constellation size $M$ are obtained from:

$$
B E R_{M}=\int_{0}^{\infty} \int_{0}^{\infty} \operatorname{BER}(M, \gamma) f(\gamma \mid \hat{\gamma}) f_{\hat{\gamma}_{\max }}(\hat{\gamma}) d \gamma d \hat{\gamma}
$$

with $\operatorname{BER}(M, \gamma)=0.2 \exp \left(\frac{-1.6 \gamma}{M-1}\right)$ for square M-QAM [21]. (11) can be reduced to: $B E R_{M}=$ $\int_{0}^{\infty} B E R(\hat{\gamma}) f_{\hat{\gamma}_{\max }}(\hat{\gamma}) d \gamma$, which is solved using (10).

\section{OPTIMAL SWITCHING BOUNDARIES}

Taking prediction errors into account, the goal is to maximize SE, which involves finding optimal switching SNR thresholds $\hat{\gamma}_{k}$ between modulation modes to satisfy instantaneous target BER $\left(B E R_{0}\right)$ and constant Power (I-BER, CP) requirement. This requires that I-BER remains below a specified error-rate level so that (8) is satisfied as $\operatorname{BER}(\hat{\gamma} ; \bar{\gamma}) \leq$ $B E R_{0}$ along with the $\mathrm{CP}$ requirement. If un-optimised switching thresholds are used for mode switching, the system will naturally degrade. The expressions of $S E$, instantaneous and average BER in the previous sections will be utilized.

\section{Instantaneous BER and Constant Power}

The average spectral efficiency or throughput of the TASP/MRC scheme can be is expanded as:

$$
B(s ; \bar{\gamma})=\sum_{k=1}^{K-1} R_{k} \int_{\hat{\gamma}_{k}}^{\hat{\gamma}_{k+1}} f_{\hat{\gamma}_{\max }}(\hat{\gamma}) d \hat{\gamma}
$$

As per the definition, a set of SNR thresholds $\mathbf{s}=$ $\left\{\hat{\gamma}_{1}, \hat{\gamma}_{2}, \ldots, \hat{\gamma}_{K}\right\}$ have to be determined at every $\bar{\gamma}$ to maximise (12) as follows:

$$
\begin{gathered}
\max _{\boldsymbol{s}} B(s ; \bar{\gamma}) \\
\text { subject to } B E R(\hat{\gamma} ; \bar{\gamma}) \leq B E R_{0}, \bar{P}=1
\end{gathered}
$$

The equality condition in (13b) should be satisfied at the rate region boundaries so that $B E R\left(\hat{\gamma}_{k} ; \bar{\gamma}\right)=B E R_{0}$. The optimal switching boundaries can thus be found which also satisfy the average transmit power constraint: $\bar{P}=1$, by inverting (8). In so doing we need to solve for $P$ which is a one time adjustment of transmit power at each $\bar{\gamma}$. To solve the two unknowns we utilize the following relation between the optimal cut off SNR level, below which data transmission is suspended (can not sustain BER), and $P$ which is:

$$
P=1 /\left[1-F_{\hat{\gamma}_{\max }}\left(\hat{\gamma}_{1}\right)\right]
$$

a value which gets close to 1 , for high $\bar{\gamma}$. Inverting (8) at the threshold boundaries, we obtain a relationship to $P$ :

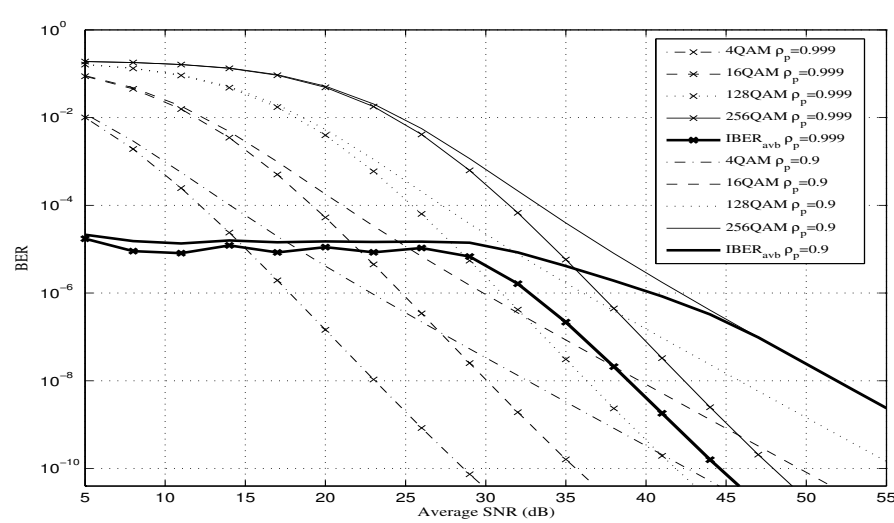

Fig. 3. Average BER, IBER $R_{a v b}$ at a target instantaneous BER of $10^{-4}$ with $\rho_{p}=0.999$ and 0.9 in a $(2 ; 1,2)$ system. Also shown are the non-adaptive average BER, $B E R_{M}$ for TASP/MRC M-QAM for the two values of $\rho_{p}$.

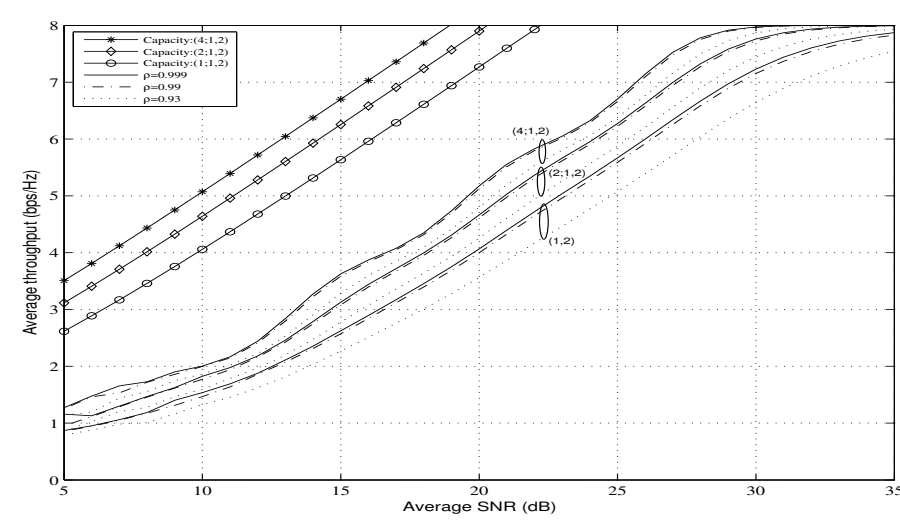

Fig. 4. Effect of $N_{t}$ and $\rho_{p}$ on $I B E R_{s e}$ (12) with $N_{r}=2$ for a target BER of $10^{-4}$; Also plotted for these setups is the maximum achievable Shannon spectral efficiency for perfect prediction in each case.

$\hat{\gamma}_{k}=-\frac{5}{8} \ln \left(\left(B E R_{0} / 0.2\right)\left(1+\mathcal{C}_{k} \cdot P\right)^{L_{r}}\right) P\left(1+\mathcal{C}_{k} \cdot P\right)\left(M_{k}-1\right)$

Finally from (14) and (15), we determine the first threshold boundary, after which $P$ itself is determined from (14). Next other boundary crossover values are obtained in turn from (15).

\section{Performance Analysis}

We choose a modulation set consisting of four square $\mathrm{M}$ QAM constellations corresponding to 2 (4-QAM), 4 (16QAM), 4 (64-QAM) and 8 (256-QAM) bits per symbol. Depending on the filter order and the amount of power of the pilot, the predictor can always be made to offer greater correlation at fixed feedback delay. For more details on how system parameters effect $\rho_{p}$ refer to [15] [22], [23]. We analyse the system performance at different values of correlation ranging from $\rho_{p}=0.999-0.9$ for different TASP setups. First we demonstrate and analyse the performance of a $(2 ; 1,2)$ TASP/MRC system with I-BER constraints and study its average BER $\left(I B E R_{a v b}\right)$ as well its $S E$. The Shannon $S E$ (4) with $\rho_{p}=1$ is also plotted to serve as a benchmark. Fig. 3 compares the performance of $I B E R_{a v b}$ for the four mode adaptive scheme calculated using (9), using the optimised thresholds of $I B E R$ calculated from (15), for 
target instantaneous BERs of $10^{-4}$ and $\rho_{p}$ as 0.999 and 0.9. Also are plotted the non-adaptive TASP/MRC schemes for the two values of $\rho_{p}$, using (11). While target BER, $B E R_{0}$, is maintained at the switching thresholds, the average BER $I B E R_{a v b}$ with I-BER constraint is always lower than $B E R_{0}$. At high average SNR, when full capacity is reached, $I B E R_{a v b}$ aligns itself with the fixed mode (non-adaptive) M-QAM BER curve of the highest modulation order, which in our case is the 256QAM. In order to see how the SE of these schemes behave with respect to increasing transmit antennas as well as $\rho_{p}$, in Fig. 4 we plot $I B E R_{s e}(12)$, for setups of $(1 ; 1,2)$, $(2 ; 1,2)$ and $(4 ; 1,2)$ for $\rho_{p}$ values of $0.999,0.99,0.93$ in each case. The target BER is the same $10^{-4}$ in each case. In each case, higher order setups increased system SE, indicating substantial power saving. We can see that the percentage drop at a specific SNR $\bar{\gamma}$ for decreasing correlation is less in higher diversity setups. However, for very poor prediction, the drop will be faster in higher order setups because of poor antenna selection. The fall in gain is more rapid in $(4 ; 1,2)$ than in $(2 ; 1,2)$, which is expected since more loss occurs from poor antenna selection. It is observed that the effect of decreasing $\rho_{p}$ was to reduce the capacity of $I B E R_{s e}$, For $\rho_{p}=0.99$, it was found that the reduction in spectral efficiencies in is very small. At lower target BERs the $I B E R_{s e}$ was found to perform worse in terms of $S E$. This effect is due to two reasons: first the cut off SNR for I-BER is increased with lower $B E R_{0}$ thereby increasing outage, resulting in a decrease in SE by disallowing transmission for a certain portion of time. As a result switching occurs for higher values of $\hat{\gamma}$, which now occurs with low probability (because of the shape of $f_{\hat{\gamma}_{\text {max }}}(\hat{\gamma})$ for small $\bar{\gamma}$. Secondly, for higher $\bar{\gamma}$, lower rate modes are selected with higher probability, thus slowing down overall increase in SE. For extreme values of delay or poor correlation, antenna selection may be ineffective and it may be better to consider only receive diversity with more resources spent on channel prediction for SNR feedback, rather than on feedback schemes or costly additional hardware at the transmitter end.

\section{CONCLUSION}

This paper has studied a multi-antenna AM diversity system employing predictive antenna selection at the transmitter and MRC at the receiver, under conditions of degraded channel knowledge. The system closed form Shannon capacity gain was first derived for optimal rate adaptation at constant power with arbitrary numbers of transmit and receive antennas, which was used as a benchmark for an instantaneous BER with optimal power constraint. While it is evident that using AM in combination with multi-antenna systems, such as TAS-MRC, can greatly increase system capacity, it becomes altogether more important to preserve these gains by ensuring good quality feedback channel information - since this conveys the switching information which is largely responsible for those gains. Incorporating channel prediction into a TAS-MRC system is shown as being pivotal in improving system performance and in maintaining that performance in the face of CSI degradation. At the same time, it is also crucial to determine optimal operating conditions for modulation mode switching which take these prediction errors into account. In doing so the system is doubly rewarded, with improved antenna selection as well as optimal mode switching. In summary, TAS-MRC is seen to be a viable approach for system implementation, even in the real-world situation of outdated channel information.

\section{REFERENCES}

[1] A. Paulraj, D. Gore, R. Nabar, and H. Bolcskei, "An overview of MIMO communications - a key to gigabit wireless," Proceedings of the IEEE, vol. 92 , no. 2, pp. $198-218$, Feb. 2004.

[2] J. Hayes, "Adaptive Feedback Communications," IEEE Trans. Commun. Technol., vol. 16, no. 1, pp. 29 -34, Feb. 1968.

[3] J. Cavers, "Variable-Rate Transmission for Rayleigh Fading Channels," IEEE Trans. Commun., vol. 20, no. 1, pp. 15 - 22, Feb. 1972.

[4] W. Webb and R. Steele, "Variable rate QAM for mobile radio," IEEE Trans. Commun., vol. 43, no. 7, pp. 2223 -2230, Jul. 1995.

[5] A. Goldsmith and P. Varaiya, "Capacity of fading channels with channel side information," IEEE Trans. Inform. Theory, vol. 43, no. 6, pp. 1986 -1992 , Nov. 1997.

[6] D. Goeckel, "Adaptive coding for time-varying channels using outdated fading estimates," IEEE Trans. Commun., vol. 47, no. 6, pp. $844-855$, Jun. 1999.

[7] A. Goldsmith and S.-G. Chua, "Variable-rate variable-power MQAM for fading channels," IEEE Trans. Commun., vol. 45, no. 10, pp. 1218 -1230 , Oct. 1997.

[8] M. Alouini and A. Goldsmith, "Adaptive modulation over Nakagami fading channels," Wireless Personal Communications, vol. 13, no. 1, pp. 119-143, May 2000.

[9] S. Falahati, A. Svensson, T. Ekman, M. Sternad et al., "Adaptive modulation systems for predicted wireless channels," IEEE Trans. Commun., vol. 52, no. 2, pp. 307-316, 2004.

[10] J. Paris, M. Aguayo-Torres, and J. Entrambasaguas, "Impact of channel estimation error on adaptive modulation performance in flat fading," IEEE Trans. Commun., vol. 52, no. 5, pp. 716 - 720, May 2004.

[11] G. Øien, H. Holm, and K. Hole, "Impact of channel prediction on adaptive coded modulation performance in Rayleigh fading," IEEE Trans. Veh. Technol., vol. 53, no. 3, pp. 758 - 769, May 2004.

[12] S. Zhou and G. Giannakis, "How accurate channel prediction needs to be for transmit-beamforming with adaptive modulation over Rayleigh MIMO channels?" IEEE Trans. Wireless Commun., vol. 3, no. 4, pp. 1285 - 1294, July 2004

[13] D. Duong and G. Oien, "Optimal Pilot Spacing and Power in RateAdaptive MIMO Diversity Systems with Imperfect CSI," IEEE Trans. Wireless Commun., vol. 6, no. 3, pp. 845 -851, Mar. 2007.

[14] Z. Chen, J. Yuan, and B. Vucetic, "Analysis of Transmit Antenna Selection/Maximal-Ratio Combining in Rayleigh Fading Channels," IEEE Trans. Veh. Technol., vol. 54, no. 4, pp. 1312 - 1321, July 2005.

[15] S. Prakash and I. McLoughlin, "Predictive Transmit Antenna Selection with Maximal Ratio Combining," in Proc. IEEE GLOBECOM 2009, Nov.30-Dec. 4 2009, pp. 1-6.

[16] J. Cavers, "An analysis of pilot symbol assisted modulation for Rayleigh fading channels [mobile radio]," IEEE Trans. Veh. Technol., vol. 40, no. 4, pp. $686-693$, Nov. 1991.

[17] W. Jakes, Microwave mobile communications. Wiley-IEEE Press, 1994.

[18] M.-S. Alouini and A. Goldsmith, "Capacity of Rayleigh fading channels under different adaptive transmission and diversity-combining techniques," IEEE Trans. Veh. Technol., vol. 48, no. 4, pp. $1165-1181$, Jul. 1999.

[19] I. Gradshteyn and I. Ryzhik, Table of integrals , Series, and Products, vol. 22.

[20] B. Choi and L. Hanzo, "Optimum mode-switching-assisted constantpower single- and multicarrier adaptive modulation," IEEE Trans. Veh. Technol., vol. 52, no. 3, pp. 536 - 560, May 2003.

[21] S. T. Chung and A. Goldsmith, "Degrees of freedom in adaptive modulation: a unified view," IEEE Trans. Commun., vol. 49, no. 9, pp. $1561-1571$, Sep. 2001.

[22] A. Duel-Hallen, S. Hu, and H. Hallen, "Long-range prediction of fading signals," IEEE Signal Processing Mag., vol. 17, no. 3, pp. 62 -75, May 2000 .

[23] T. Ekman, "Prediction of mobile radio channels, modeling and design," Ph.D. dissertation, Uppsala University, Sweden, 2002. 